\title{
KATP channel is novel targets for Alzheimer's disease therapeutics
}

\section{Shigeki Moriguchi, Kohji Fukunaga}

\author{
Department of Pharmacology, Graduate School of Pharmaceutical Sciences, Tohoku University, Japan
}

Memantine ameliorates progressive symptomes in Alzheimer's disease (AD) through moderate inhibition of N-methyl-Daspartate receptors (NMDARs). Here we report that a novel target of mementine, ATP-sensitive K+ (KATP) channels are implicated in memory improvement. KATP channels Kir6.1 or Kir6.2 are composed with sulfonylurea receptors (SURs), which are distributed both in peripheral tissues and central nervous system. We confirmed that memantine improves both memory impairment and perturbed NMDAR-dependent LTP in APP23 mouse hippocampus. Unexpectedly, memantine in vivo increased CaMKII activity in APP23 hippocampus, and memantine-induced enhancement of hippocampal LTP and CaMKII activity was in vitro abolished by treatment with pinacidil, a specific opener of KATP channels. We therefore confirmed that memantine inhibits KATP channels Kir6.1 and Kir6.2 and elevates intracellular $\mathrm{Ca} 2+$ concentrations by inhibition of Kir6.1 or Kir6.2. Kir6.2 was preferentially expressed in the postsynaptic regions, whereas Kir6.1 was predominant in mouse hippocampal neuron dendrites. Finally, we confirmed that Kir6.2 heterozygous mutant mice exhibit severe memory deficits and hippocampal LTP impairment that could not be rescued by memantine administration. Taken together, we propose a novel strategy that memantine inhibits Kirs 6.2/6.1 activities, thereby improving memory impairment in $\mathrm{AD}$ patients. 